\title{
Butyric acid-producing anaerobic bacteria as a novel probiotic treatment approach for inflammatory bowel disease
}

Inflammatory bowel disease (IBD), of which Crohn's disease (CD) and ulcerative colitis (UC) are the most common manifestations, is characterized by chronic inflammation of the lining of the gastrointestinal tract, which causes severe watery and bloody diarrhoea, and abdominal pain. IBD is often debilitating and is characterized by onset at a young age and extra-intestinal manifestations. Whereas CD can affect any part of the gastrointestinal tract, UC is usually confined to the colon and rectum. IBD is an emerging disease and the incidence amounts to 20/100 000 in Europe and North America. There is a link with the social and economic development of the countries: the increase in IBD was first seen in northern Europe and North America, followed by the rest of Europe, Japan, South America and certain parts of Asia (Cohen, 2000; Ouyang et al., 2005). Although the exact aetiopathogenesis of IBD are not clear, it is widely accepted that the disease derives from an inappropriate immune response in genetically susceptible individuals as the result of a complex interaction between environmental factors, the microbiota and the intestinal immune system (Danese \& Fiocchi, 2006).

In IBD, the response to conventional drug therapy is suboptimal. This therapy is associated with a number of disadvantages, including a considerable burden of side effects, and surgery is still required in a subgroup of patients. Because IBD represents a distortion of the relationship between the gut microbiota and the host response, probiotics and prebiotics have been proposed as possible therapeutic products. The probiotics that are being tested for treatment of IBD are mainly lactic acid bacteria, i.e. lactobacilli and bifidobacteria. Although a decrease in the number of lactic acid bacteria has been shown in IBD, administration of lactic acid bacteria has yielded inconsistent results with respect to the treatment of IBD
(Gueimonde et al., 2007; Hedin et al., 2007). The only well-documented indication for the use of probiotics in IBD is in the treatment of pouchitis, particularly using a mixed lactobacillibifidobacteria product (Mimura et al., 2004). Many trials using probiotics either as maintenance therapy in $\mathrm{UC}$, to treat active $\mathrm{UC}$, to maintain remission in $\mathrm{CD}$, or to treat active $\mathrm{CD}$, have yielded mixed results, concluding that their use cannot be recommended based on the available evidence (Hedin et al., 2007).

Butyric acid, produced within the intestinal lumen by bacterial fermentation of dietary carbohydrates, exerts a wide variety of effects on intestinal function (Hamer et al., 2008). First of all, butyric acid is the preferred source of energy for colonocytes. It affects cellular proliferation, differentiation and apoptosis. Moreover, butyric acid has well documented antiinflammatory effects. Inhibition of histone deacetylase activity, resulting in hyperacetylation of histones, and as a consequence suppression of nuclear factorkappa B activation, is a likely explanation. Secondly, it has been proposed that butyric acid reinforces the colonic defence barrier by increasing production of mucins and antimicrobial peptides. Thirdly, it has been shown that butyric acid decreases intestinal epithelial permeability by increasing the expression of tight junction proteins. Antiinflammatory activities, combined with a strengthening of the mucosal barrier integrity, are ideal properties for therapeutic compounds against IBD-like syndromes. Indeed it has been shown that butyrate enemas can yield positive results in the treatment of active UC (Breuer et al., 1997).

Butyric acid thus has a potential therapeutic value against IBD, but the actual delivery into the gut is still problematic. Several mechanisms are possible.

(a) Fermentation of dietary fibre leads to butyrate production. An increase in butyrate production may result from a direct stimulation of butyrate producers or indirect effects such as metabolic cross-feeding of fermentation products from other bacterial groups (Flint et al., 2007).

(b) The use of butyric acid-coated tablets is a possible delivery method, but the tablets may not always release their content at the intended location because of interindividual differences in gut lumen $\mathrm{pH}$ and transit time. Moreover the taste is very unpleasant. Alternative approaches are the consumption of butyrylated starch and the use of butyryl-L-carnitine as a potential prodrug (Srinivas et al., 2007; Bajka et al., 2008).

(c) Rectal butyric acid enemas hold promise but the use of enemas is often hampered by a low compliance rate, and a short and discontinuous exposure of the colon mucosa to the butyrate (Breuer et al., 1997).

(d) A final possibility is the administration of colonizing butyric acid-producing bacteria, enabling in situ production of butyric acid.

Several studies have shown that, numerically, the majority of butyrateproducing bacteria found in human faeces are highly oxygen-sensitive anaerobes belonging to the clostridial clusters IV and XIVa (Louis \& Flint, 2009). Decreases in members of the butyrate-producing clostridial clusters IV and XIVa have been reproducibly reported in the gut of IBD patients (Sokol et al., 2008; Takaishi et al., 2008). These species are thus probiotic candidates for the treatment of IBD. Recently, considerable progress has been made in the isolation of these strictly anaerobic butyric acid-producing bacteria from the human gut. It has been shown 
that lactic acid, produced in vitro by lactic acid bacteria, is used by some strictly anaerobic butyrate-producing bacteria of clostridial cluster XIVa for the production of high concentrations of butyric acid (Louis \& Flint, 2009). This mechanism is called cross-feeding and could explain why administration of lactic acid bacteria to IBD patients can in certain cases be an advantage, i.e. through stimulation of butyric acid production. However, other possible mechanisms of action, including immunoregulatory effects, also have been suggested (Geier et al., 2007). An ideal probiotic would thus be a colonizing bacterium that combines systemic antiinflammatory and immunoregulatory effects with delivery of high butyrate levels at the site of action and that can be ingested in a stable form, such as spores. One potential problem is that bowel conditions in IBD patients may not be optimal to support growth of these bacterial species. However, butyrate producers present in the colon are phylogenetically diverse and it has not been investigated in detail yet which species are decreased in IBD. Therefore, it is likely that strains can be identified that are good colonizers. Furthermore, a synbiotic approach, using a prebiotic in combination with a butyrate producer, may help to reestablish such bacteria in the individual in the diseased state. First attempts have been made in animal models of IBD to apply butyrate-producing bacteria (Sokol et al., 2008; Eeckhaut et al., 2009). Strains of clostridium cluster IV and XIVa were found to be highly successful in decreasing inflammation and necrosis in rodent IBD models. Indeed, Faecalibacterium prausnitzii, a species belonging to cluster IV, has been shown to be much less prevalent in the gut microbiota of IBD patients. This species showed great promise in counterbalancing dysbiosis in a mouse IBD model (Sokol et al., 2008, 2009). Interestingly, components of the culture supernatant of this bacterium had the capacity to decrease production of the inflammatory cytokines interleukin 12 and gamma interferon, and to increase production of the anti-inflammatory cytokine interleukin 10, in peripheral blood mononuclear cells in a way independent of butyrate (Sokol et al., 2008). Oral and intraperitoneal administration of the culture supernatant in a mouse IBD model reduced colitis and mortality, respectively
(Sokol et al., 2008). This clearly suggests that F. prausnitzii secretes certain molecules that have the potential to be used as therapeutics. In fact it has not been resolved whether butyrate is the key molecule in the anti-colitis effects of $F$. prausnitzii or whether these depend on a combination of secreted compounds yet-to-be identified that might act independently or together with butyrate. Notably, however, $F$. prausnitzii administration led to a shift in the microbiota composition that could also have complex effects on colitis (Sokol et al., 2008). It has been shown that the inflamed intestinal mucosa of IBD patients has a defective butyrate metabolism, leading to reduced efficiency of butyrate, again suggesting that other compounds in addition to butyrate may be involved in the beneficial effects of the butyrate-producing microbiota (Thibault et al., 2010). Nevertheless, it is clear that the pool of butyrate-producing bacteria from clostridial cluster IV and XIVa harbours very promising preventive and therapeutic probiotic strains. In a comparative study using four strains, it was shown that a particular strain from clostridium cluster IV, Butyricicoccus pullicaecorum, was superior in decreasing lesions in a rat IBD model compared to F. prausnitzii (Eeckhaut et al., 2009). It is therefore suggested that research efforts should be increased to test the therapeutic value of these next generation probiotic strains, and to identify further strains that are able to colonize the gut of IBD patients without being outcompeted.

\section{Filip Van Immerseel, ${ }^{1}$ \\ Richard Ducatelle, ${ }^{1}$ Martine De Vos, ${ }^{2}$ Nico Boon, ${ }^{3}$ Tom Van De Wiele, ${ }^{3}$ Kristin Verbeke, ${ }^{4}$ Paul Rutgeerts, ${ }^{4}$ Benedikt Sas, ${ }^{5}$ Petra Louis ${ }^{6}$ and Harry J. Flint ${ }^{6}$}
${ }^{1}$ Department of Pathology, Bacteriology and Avian Diseases, Faculty of Veterinary Medicine, Ghent University, Ghent, Belgium
${ }^{2}$ Department of Gastroenterology, Ghent University Hospital, Ghent, Belgium
${ }^{3}$ Laboratory of Microbial Ecology and Technology (LabMET), Faculty of Bioscience Engineering, Ghent University, Ghent, Belgium
${ }^{4}$ Department of Gastroenterology and Leuven Food Science and Nutrition

Research Centre (LFoRCe), University Hospital, Leuven, Belgium

${ }^{5}$ Centre of Excellence Food2Know, Ghent University, Ghent, Belgium

${ }^{6}$ Microbial Ecology Group, Gut Health Programme, Rowett Institute of Nutrition and Health, University of Aberdeen, Aberdeen, UK

Correspondence: Filip Van Immerseel (filip.vanimmerseel@ugent.be)

Bajka, B. H., Clarke, J. M., Cobiac, L. \& Topping, D. L. (2008). Butyrylated starch protects colonocyte DNA against dietary proteininduced damage in rats. Carcinogenesis 29, 2169-2174.

Breuer, R. I., Soergel, K. H., Lashner, B. A., Christ, M. L., Hanauer, S. B., Vanagunas, A., Harig, J. M., Keshavarzian, A., Robinson, M. \& other authors (1997). Short-chain fatty acid rectal irrigation for left-sided ulcerative colitis: a randomized, placebo-controlled trial. Gut 40, 485-491.

Cohen, M. L. (2000). Changing patterns of infectious disease. Nature 406, 762-767.

Danese, S. \& Fiocchi, C. (2006).

Etiopathogenesis of inflammatory bowel diseases. World J Gastroenterol 12, 4807-4812.

Eeckhaut, V., Flahou, B., Romero, C., Van Der Heyden, S., Pasmans, F., Haesebrouck, F., Ducatelle, R. \& Van Immerseel, F. (2009). The anaerobic butyrate-producing strain Butyricicoccus pullicaecorum decreases colonic inflammation and ulceration in a TNBS-induced colitis rat model. In 5th Probiotics, Prebiotics and New Foods Congress, Rome, Italy. http:// www.probiotics-prebiotics-newfood.org/pdf/ 5th_Probiotics_Prebiotics_Newfood.pdf

Flint, H. J., Duncan, S. H., Scott, K. P. \& Louis, P. (2007). Interactions and competition within the microbial community of the human colon: links between diet and health. Environ Microbiol 9, 1101-1111.

Geier, M. S., Butler, R. N. \& Howarth, G. S. (2007). Inflammatory bowel disease: current insights into pathogenesis and new therapeutic options; probiotics, prebiotics and synbiotics. Int J Food Microbiol 115, 1-11.

Gueimonde, M., Ouwehand, A., Huhtinen, H., Salminen, E. \& Salminen, S. (2007). Qualitative and quantitative analyses of the bifidobacterial microbiota in the colonic mucosa of patients with colorectal cancer, diverticulitis and inflammatory bowel disease. World J Gastroenterol 13, 3985-3989.

Hamer, H. M., Jonkers, D., Venema, K., Vanhoutvin, S., Troost, F. J. \& Brummer, R. J. (2008). Review article: the role of butyrate on colonic function. Aliment Pharmacol Ther 27, 104-119.

Hedin, C., Whelan, K. \& Lindsay, J. O. (2007).

Evidence for the use of probiotics and prebiotics 
in inflammatory bowel disease: a review of clinical trials. Proc Nutr Soc 66, 307-315.

Louis, P. \& Flint, H. J. (2009). Diversity, metabolism and microbial ecology of butyrateproducing bacteria from the human large intestine. FEMS Microbiol Lett 294, 1-8.

Mimura, T., Rizzello, F., Helwig, U., Poggioli, G., Schreiber, S., Talbot, I. C., Nicholls, R. J., Gionchetti, P., Campieri, M. \& Kamm, M. A. (2004). Once daily high dose probiotic therapy (VSL\#3) for maintaining remission in recurrent or refractory pouchitis. Gut $\mathbf{5 3}$, 108-114.

Ouyang, Q., Tandon, R., Goh, K. L., Ooi, C. J., Ogata, H. \& Fiocchi, C. (2005). The emergence of inflammatory bowel disease in the Asian
Pacific region. Curr Opin Gastroenterol 21, 408-413.

Sokol, H., Pigneur, B., Watterlot, L., Lakhdari, O., Bermudez-Humaran, L. G., Gratadoux, J. J., Blugeon, S., Bridonneau, C., Furet, J. P. \& other authors (2008). Faecalibacterium prausnitzii is an anti-inflammatory commensal bacterium identified by gut microbiota analysis of Crohn disease patients. Proc Natl Acad Sci U S A 105, 16731-16736.

Sokol, H., Seksik, P., Furet, J. P., Firmesee, O., Nion-Larmurier, I., Beaugerie, L., Cosnes, J., Corthier, G., Marteau, P. \& Doré, J. (2009). Low counts of Faecalibacterium prausnitzii in colitis microbiota. Inflamm Bowel Dis 15, 1183-1189.

Srinivas, S. R., Prasad, P. D., Umapathy, N. S., Ganapathy, V. \& Shekhawat, P. S. (2007).
Transport of butyryl-L-carnitine, a potential prodrug, via the carnitine transporter OCT2 and the amino acid transporter $\mathrm{ATB}^{0,+}$. Am J Physiol Gastrointest Liver Physiol 293, G1046-G1053.

Takaishi, H., Matsuki, T., Nakazawa, A., Takada, T., Kado, S., Asahara, T., Kamada, N., Sakuraba, A., Yajima, T. \& other authors (2008). Imbalance in intestinal microflora constitution could be involved in the pathogenesis of inflammatory bowel disease. Int J Med Microbiol 298, 463-472.

Thibault, R., Blachier, F., Darcy-Vrillon, B., De Coppet, P., Bourreille, A. \& Segain, J. P. (2010). Butyrate utilization by the colonic mucosa in inflammatory bowel diseases: a transport deficiency. Inflamm Bowel Dis $\mathbf{1 6}$ (in press). 